\title{
MULTIDIMENSIONAL FLOW MAPPING FOR PROPORTIONAL VALVES
}

\author{
André Sitte*, Oliver Koch, Jianbin Liu, Ralf Tautenhahn, Jürgen Weber \\ Institut für Mechatronischen Maschinenbau, Technische Universität Dresden, Helmholtzstrasse $7 a, 01069$ Dresden \\ * Corresponding author: Tel.: +49 351 46333707; E-mail address: andre.sitte@tu-dresden.de
}

\begin{abstract}
Inverse, multidimensional input-output flow mapping is very important for use of valves in precision motion control applications. Due to the highly nonlinear characteristic and uncertain model structure of the cartridge valves, it is hard to formulate the modelling of their flow mappings into simple parameter estimation problems. This contribution conducts a comprehensive analysis and validation of three- and four-dimensional input-output-mapping approaches for a proportional pilot operated seat valves. Therefore, a virtual and a physical test-rig setup are utilized for initial measurement, implementation and assessment. After modeling and validating the valve under consideration, as a function of flow, pressure and temperature different mapping methods are investigated. More specifically, state of the art approaches, deep-learning methods and a newly developed approach (extPoly) are examined. Especially ANNs and Polynomials show reasonable approximation results even for more than two inputs. However, the results are strongly dependent on the structure and distribution of the input data points. Besides identification effort, the invertibility was investigated.
\end{abstract}

Keywords: Valve Control, Flow Mapping, Polynomial Fitting, Artificial Neural Networks, Deep Learning

\section{INTRODUCTION}

Modern electrohydraulic drive and control tasks are subject to high demands in terms of flexibility and complexity. Various fields of application can be named such as energy-efficient operation, high-precision, closed-loop-control-tasks, automation, fault detection, condition monitoring and diagnostics. The majority of today's hydraulic drive systems incorporate valveoperated actuators. Especially independent metering control systems enable energy efficient and high performance operation by decoupling meter-in and meter-out flow, using regeneration flow and enhanced control possibilities of multiple input system structures. The key elements of such systems are individual valves, preferably of seat type due to advantageous dynamic and static properties. Since the mathematical models of cartridge valves flow mapping is complex, it cannot be described by typical analytical nonlinear equations, commonly used.
Main challenges are the complex non-linear characteristics of hydraulic components, which are exposed to a wide range of external environmental and operational effects as well as internal factors like aging. Besides mainly oilrelated thermal dependencies, in particular flowrelated friction and throttle losses determine the static and dynamic input-output-behavior.

A common strategy to compensate for these nonlinearities is an inverse feedforward controller. Usually nonlinear or unknown physical relations are modelled using empirical coefficients measured at typical operating points. In many cases, the achievable accuracy is insufficient but the experimental and computational effort to raise the mapping-quality increases dramatically with higher number of measuring points. The present paper aims to analyze different approaches for (inverse) inputoutput mapping of a pilot operated seat valve. 


\section{STATE OF THE ART}

Experimental system identification, in particular the measurement of input-output-signals, has low information content regarding internal processes and parameter dependencies. A distinction between parametric models utilizing explicit parameters within mathematical/physical equations and nonparametric models, using implicit parameters with purely functional relationships of behavior, can be made [1]. In industrial applications, nonparametric models are widespread. Common to all of them is the problem of identifying and implementing threeor higher-dimensional representations as a nonlinear mapping of two (or more) input variables $x_{i n}, y_{i n}, i_{i n}$ to an output variable $z_{\text {out }}=$ $f\left(x_{i n}, y_{i n}, i_{i n}\right)$. Among others, the following methods can be found: 1. grid maps, 2 . polynomial approximations, and 3 . artificial neural networks.

Nonlinear static relationships with grid maps are widespread because they are easy to implement on Electronic Control Units (ECU). Often, equidistant grids with $(m \cdot n)$ supporting points in combination with surface interpolation methods are applied. Since each grid-point must correspond to a measuring point, the quality of the map significantly depends on the number of nodes and the degree of non-linearity. Due to computational effort, the number of dimensions is limited. The second class of approximation methods are higher order polynomials such as algebraic polynomials, orthogonal polynomials or Chebyshev polynomials. An advantage of this type of models is a compact form of mathematical representation and an efficient calculation mode. The system degree determines shape and order of the polynomial. With the help of regression methods, the unknown polynomial coefficients are identified and parameterized [2]. Second order polynomials are usually easy to invert analytically, whereas functions of higher order require a numerical solution. Application examples for compensating methods can be found in [3], [4]. Artificial neural networks are highly capable of approximating and representing complex nonlinear and high dimensional functions [5]. There are different classes of neural networks and corresponding training algorithms, each suitable for different approximation problems. Typical examples are Multi-Layer-
Perceptrons (MLP) or Radial Base Functions (RBF) [6], [7], [8].

\section{IDENTIFICATION METHODS}

\subsection{General aspects}

Experimental modelling (identification) of measurement data enables the determination of characteristic maps. Figure 1 shows the proposed workflow.

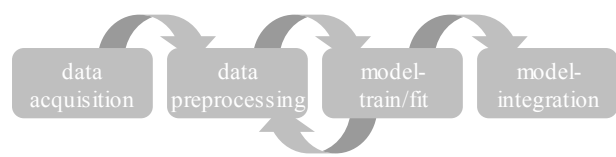

Figure 1: Characteristic map identification workflow

The first step is to acquire a set of measurement data representing all dependencies of interest. It is crucial to collect this data under varying operating conditions and measuring regimes.

If the measurement data is limited or insufficient, simulation data may be used instead or as supplement. This data is referred to as synthetic data. A combination of synthetic and sensor data may be a reasonable compromise.

The next step of the workflow is preprocessing of the raw data. Typically missing or faulty values, noise and outliers are processed and set up for further steps. In some cases a scaling is beneficial, since big values generate great residuals. This is equivalent to weighting in advance.

The following task is finding and training (fitting) the model that captures the relationship between the input and output. Starting point of the experimental modeling is the determination of a suitable model structure. The corresponding model parameters are adapted using optimization procedures, to match input/output behavior of model and measured data. The selected model structure must allow mapping for all physical effects of interest. Basically linear and non-linear as well as static and dynamic models may be distinguished. This contribution deals with static, nonlinear models. In general, nonlinear mapping $f$ of two input variables $x_{i n}$ and $y_{\text {in }}$ is formulated by:

$$
z_{\text {out }}=f\left(x_{\text {in }}, y_{\text {in }}\right)
$$

To optimize the model parameters usually the sum square error between the measured output $\mathrm{z}$ and estimated output $\hat{z}$ is minimized [9]. 


$$
R(\theta)=\sum_{i=1}^{n}(z(i)-\hat{z}(i))^{2}
$$

The final step is the integration (deployment) of the model into the control platform. Memory requirements as well as cycle time of the device may potentially affect the quality of the model results.

According to [9] the generation of grid maps from measurement data can be performed in three ways. The approaches are adoptable and valid for many identification procedures.

Method 1: The definition of the grid lines/points is either equidistant or it can be chosen individually (optimal distribution) considering process knowledge. The intersections of the grid lines provide direct estimates or training points. The procedure does not require any optimization.

Method 2: As in method 1, an a-priori definition of a grid points takes place. Using optimization techniques the optimal position of a grid point from arbitrarily distributed measurement data is performed in a postprocessing step. A necessary condition is the availability of a sufficient number of data points.

Method 3: [10] proposes a method for optimization of grid point positions and heights at the same time. Here nonlinear optimization methods are necessary.

\subsection{Grid based look up tables - gridLUT}

Grid-based look-up tables are the most common type of nonlinear static models used in practice [9], [2]. A nonlinear relationship is modelled by storing input-data-tuples $\left[\mathrm{x}_{\mathrm{i}}, \mathrm{y}_{\mathrm{i}}\right]$ in a grid.
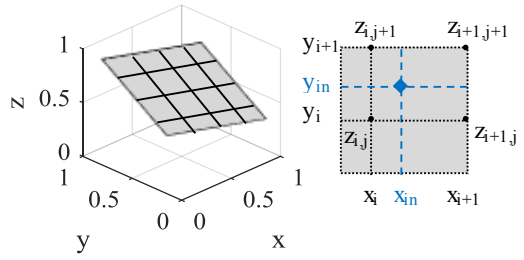

Figure 2: Areas and grid points used for surface interpolation (gridLUT)

Typically, they are limited to one or twodimensional implementations, because measuring approaches mostly consider one effect at a time for a corresponding output. Interpretation and manipulation of 1D-data is very easy and user-friendly.
The model output $\mathrm{z}$ for an input vector $[\mathrm{x}, \mathrm{y}]$ is calculated by local interpolation between the surrounding nodes. In 1D-case, linear interpolation methods may be used. For 2D-case, bilinear interpolation between four surrounding points (vertex points) is necessary. At grid-point positions, the model output is equal to the measured data. Intermediate values that lie within the grid are calculated with aforementioned surface interpolation method [6].

$\mathrm{z}=\left[z_{i, j}\left(x_{i+1}-\mathrm{x}\right)\left(y_{i+1}-\mathrm{y}\right)+\right.$ $z_{i+1, j}\left(\mathrm{x}-x_{i}\right)\left(y_{i+1}-\mathrm{y}\right)+z_{i, j+1}\left(x_{i+1}-\right.$ $\left.\mathrm{x})\left(\mathrm{y}-y_{j}\right)+z_{i+1, j}\left(\mathrm{x}-x_{i}\right)\left(\mathrm{y}-y_{j}\right)\right] /$ $\left[\left(x_{i+1}-x_{i}\right)\left(y_{j+1}-y_{j}\right)\right]$

To determine a desired point $\left[\mathrm{x}_{\mathrm{in}}, \mathrm{y}_{\text {in }}\right]$ in the input space, all the heights $\mathrm{z}$ of the four surrounding grid-points must be explicitly known. The heights of the four vertices are multiplied by the opposing areas and then divided by the total area value.

The number of grid-points in each dimension are chosen according to the accuracy demanded. However, the number of data point's $\mathrm{n}_{\mathrm{P}}$ grows exponentially with the number of input dimensions $\mathrm{k}$ :

$$
n_{p}=\prod_{i=1}^{k} n_{i}
$$

If measurement data points are incomplete or do not correspond to the desired positions of the grid, estimation techniques or extra measuring activities have to considered to derive additional grid points. Additional efforts and costs are to be expected. An arbitrary and optimal positioning of grid points represents a nonlinear optimization problem, which potentially increases the resulting quality of the method.

\subsection{Polynomials}

\section{Algebraic polynomials - algPoly}

Polynomials are typical approximators, characterized in particular by a high calculation and evaluation speeds [1]. The choice of the maximum degree depends on approximation accuracy and other factors. With the help of an algebraic polynomial $z$ a function $f$ is approximated as follows: 


$$
\begin{aligned}
z(x, y)=a_{0,0} & +a_{1,0} x+a_{0,1} y+a_{2,0} x^{2} \\
& +a_{0,2} y^{2}+a_{1,1} x y \\
& +a_{m, 0} x^{m}+a_{m-1,1} x^{m-1} \\
& +a_{1, m-1} y^{m-1}+a_{0, m} y^{m}
\end{aligned}
$$

Polynomial terms $(\mathrm{x}, \mathrm{y})$ are multiplied by the coefficients $a_{i, j}$, which represent the free parameters of the model approach. With the maximum degree $k$ of the polynomial, the number $n_{C}$ of unknown coefficients $a_{i, j}$ is:

$$
n_{C}=\frac{1}{2}(k+1)(k+2)
$$

There is an uncertainty regarding the order of the polynomials. It should not exceed $\left(n_{p}-1\right)$, where $n_{p}$ is the number of data points per input. High order polynomials show an increased oscillation behaviour. For practical use $3^{\text {rd }}$ to $6^{\text {th }}$ order, including interaction-terms, are suitable. Generally speaking, polynomials are characterized by a very good smoothness, simple parameter determination but limited extrapolation capabilities [9].

\section{Semi empirical flow mapping - twoLine}

In [4] Huhtala proposed a mapping description as a function of system variables. The method incorporates the variation of one parameter while the other remains constant. This results in two curves, which are obtained by polynomial fitting. The measurement data needs to cover the whole range of the operating field. The flow rate through a valve can be described as a function of pressure drop and input signal. The basis of the so-called "two-line-models" are measured data of the test valve near the operating boundaries. First, the input voltage is kept constant and the pressure drop is varied from min to max. Next, the input voltage is kept constant at max and the pressure drop dependency is measured. With help of these two data sets, the flow rate as a function of pressure drop can be obtained by using polynomial fitting:

$$
z_{i}(y)=a_{i, 1} e^{a_{i, 2} y}-a_{i, 3} e^{-a_{i, 4} y}
$$

Next step is a min-max normalization:

$$
\hat{z}_{i}(y)=\frac{z_{i}(y)-z_{i}\left(y_{\min }\right)}{z_{i}\left(y_{\max }\right)-z_{i}\left(y_{\min }\right)}
$$

The curves for output $\mathrm{z}$ (flow rate) as a function of input $\mathrm{x}$ (voltage) are identified in the previous mentioned way. First the input y (pressure drop) is kept constant at min (resp. max) and the input voltage is varied from min to max. With help of the polynomial fitting, the flow rate as a function of input voltage can be obtained. The flow rate as a function of pressure drop and input voltage is:

$$
\begin{gathered}
z(x, y)=\left\{\left[\hat{z}_{2}(y)-\hat{z}_{1}(y)\right]\left(\frac{x-x_{\min }}{x_{\max }-x_{\min }}\right)\right. \\
\left.+\hat{z}_{1}(y)\right\} \cdot\left[z_{4}(x)-z_{3}(x)\right] \\
+z_{3}(x)
\end{gathered}
$$

Figure 3 depicts the working principle of the two-line-method (twoLine).

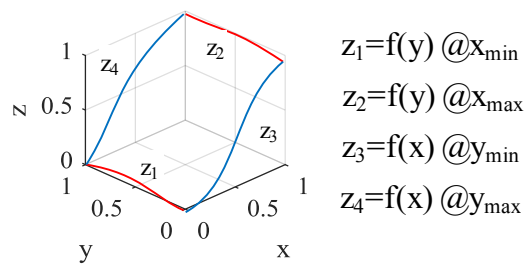

Figure 3: Principle of operation of two-line-method

III Extended algebraic polynomials - extPoly

The proposed polynomial approximation uses the following general approach:

$Y=\sum_{i_{0}=0}^{m_{0}} \sum_{i_{1}=0}^{m_{1}} \ldots \sum_{i_{n}=0}^{m_{n}} a_{i_{0} i_{1} \cdots i_{n}} Y_{0}^{i_{0}} Y_{1}^{i_{1}} \ldots Y_{n}^{i_{n}}$

The n-dimensional output $Y$ is a result of a polynomial combination of 1-dimensional functional approximation $Y_{n}$ for each dimension. For this paper, $Y_{k}$ represents a char curve with its sampling point tuples $\left(\hat{x}_{k}, \hat{y}_{k}\right)$ :

$$
Y_{k}=f_{c}\left(\hat{x}_{k}, \hat{y}_{k}, x_{k}\right)
$$

The char curve uses the linear interpolation:

$$
Y_{k}=\frac{\hat{y}_{i+1}-\hat{y}_{i}}{\hat{x}_{i+1}-\hat{x}_{i}}\left(x_{k}-\hat{x}_{i}\right)
$$

For instance, a simple three-dimensional relationship has the specific approach:

$$
Y=a_{00}+a_{10} Y_{0}+a_{01} Y_{1}+a_{11} Y_{0} Y_{1}
$$

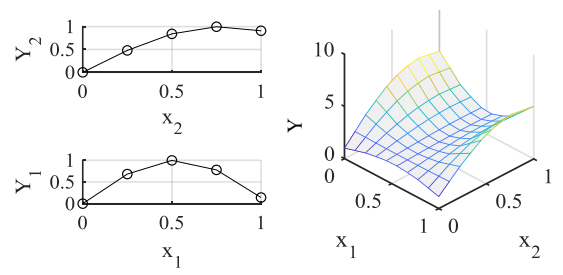

Figure 4: Polynomial approximation example (extPoly) 
Figure 4 exemplarily illustrates a result for a surface based on 24 parameters (10 for each dimension and the four polynomial factors $a_{i k}$ ). This approach is advantageous in the software development for embedded systems to integrate multi-dimensional dependencies with a reduced set of parameters. Using a gradient-based optimization finds the parameters for each approximation.

\subsection{Artificial neural networks - ANN}

Artificial neural networks became very popular in the context of Machine Learning and Big-Data during the last years. Nevertheless, their foundation was laid up back in the 1940s already [11]. Since then a broad variety of different network architectures was developed. However, they all share a common working principle:

The main idea is to connect the outputs and inputs of some information processing units (called nodes or neurons) and to modify their sensitivity to each other by weighting and biasing these connections. Since the nodes and their links among each other are quite similar to the nerve cells (called neurons) and their connections by axons and dendrites the name "artificial neural networks" (ANN) was given.

According to [12] different switching functions inside the information processing units (e.g. binary, linear or sigmoid), different mechanisms for the parametrization of the weights (e.g. supervised, unsupervised and reinforcement learning) and different network architectures (e.g. feed forward, Kohonen or Hopfield networks) for the connections between the units may be used depending on the application. Typical applications are classification, function approximation, image processing or speech recognition. For function approximation multilayer perceptrons (MLPs) or radial basis function networks are advised in literature.

The weights and biases of MPLs are usually parametrised from known data (inputs and associated outputs) in a specialised optimization process, which is called backpropagation training. Afterwards the resulting network will produce similar outputs according to the inputs it was "trained" for. Nevertheless, this concept also provides some generalization. To a certain extent even previously unknown inputs will result in an appropriate output if the network represents the underlying correlations correctly [13].

In the following investigation Bayesian Regularization backpropagation [14] was used for networks with two hidden layers (each holding between two and eight neurons). In this case the output of a single neuron of the $\mathrm{n}^{\text {th }}$ layer is

$$
\sigma_{n j}=\varphi\left(\boldsymbol{w}_{n j}^{T} \cdot \boldsymbol{x}_{j}+\theta_{n j}\right) .
$$

Using following expression for the weight factor

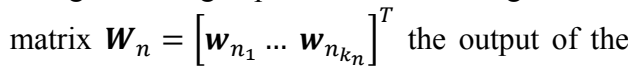
whole network results from the matrix equation

$$
y=\varphi\left(\boldsymbol{W}_{n} \cdot \varphi\left(\boldsymbol{W}_{n-1} \cdot \varphi+\boldsymbol{\theta}_{n-1}\right)+\boldsymbol{\theta}_{n}\right)
$$

with the bias vector $\boldsymbol{\theta}_{\boldsymbol{n}}=\left[\theta_{n_{1}} \ldots \theta_{n_{k_{n}}}\right]^{\boldsymbol{T}}$ for each layer number $n$. The number of neurons in layer $l$ is $n_{n}$ and the switching function

$$
\varphi(\boldsymbol{x})=2 /(1+\exp (-2 \cdot \boldsymbol{x}))-1
$$

is applied element wise and could be substituted by any another sigmoid function.

\section{APLLICATION AND MODELLING}

\subsection{Proportional flow control valve}

One example for a seat type proportional flow control valve is depicted in Figure 5. The operating principle of the so-called Valvistor (type A - proportional flow from $\mathrm{p}_{1}$ to $\mathrm{p}_{2}$ ) is based on flow feedback for displacement control of the main poppet (MP- (3))
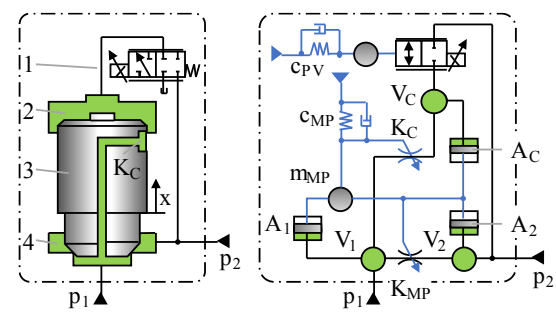

Figure 5: Seat type proportional valve schematic (left)/ Simulation model structure (right)

Due to a negative overlap of the control orifice, the pressure $\mathrm{p}_{\mathrm{C}}$ in the control chamber $\mathrm{V}_{\mathrm{C}}$ is equal to the pressure $p_{1}$ at the valve inlet $V_{1}$. Area $A_{C}$ is greater than area $A_{1}$, ensuring a closing of the main poppet. Opening the pilot valve (1), enables the pilot flow $\mathrm{QPV}_{\mathrm{PV}}$ and reduces the control pressure $\mathrm{p}_{\mathrm{C}}$ in the control chamber. The main 
poppet starts moving until the equilibrium of forces is established. Neglecting flow- and friction forces and rearranging the force balance equation for the main poppet leads to:

$$
p_{C}=\frac{p_{1}}{\varphi}+\left(\frac{\varphi-1}{\varphi}\right) p_{2}, \text { with } \varphi=\frac{A_{1}+A_{2}}{A_{1}}
$$

If the area ratio $\varphi=2$, the pressure drop $\Delta p_{1 C}=$ $p_{1}-p_{C}$ across control-orifice is equivalent to the pressure drop $\Delta p_{C 2}=p_{C}-p_{2}$ across the pilot valve. The flow rate across control-orifice results in:

$$
Q_{C}=Q_{P V}=K_{C}\left(x_{0}+x\right) \sqrt{\Delta p_{1 C}}
$$

According to eq. (17) and (18), the following interrelation can be obtained:

$$
x=\left(\frac{Q_{P V}}{K_{C}} \sqrt{\frac{(\varphi-1)}{\varphi\left(p_{1}-p_{2}\right)}}\right)-x_{0}
$$

The flow rate across main poppet is denoted as:

$$
Q_{M P}=K_{M P} x \sqrt{\Delta p_{12}}
$$

Neglecting the negative overlap $\mathrm{x}_{0}$ in eq. (19) and substituting eq. (19) for eq. (20), results in following equation:

$$
Q_{M P}=\left(\frac{K_{M P}}{K_{C}} \sqrt{\frac{\varphi-1}{\varphi}}\right) Q_{P V}
$$

The total flow rate is:

$Q_{T}=Q_{M P}+Q_{P V}$

The "Valvistor" is similar to a transistor, which amplifies a small current through the pilot circuit. Amongst others, more information can be obtained in [15] or [16].

\subsection{Experimental setup and results}

To evaluate the static and dynamic characteristics of the proportional seat-type valve, a test bench has been set up. Figure 6 briefly illustrates the experimental setup, including pump, sensors and the loading valve controlling the pressure drop across the test valve. A displacement sensor is installed on the main poppet as well as on the pilot spool. The test rig setup is restricted to $\mathrm{Q}_{\max }=2001 / \mathrm{min}$ and $\mathrm{p}_{\max }=200 \mathrm{bar}$. The test valve is set to discrete opening values. Varying the outlet pressure, the oil flow through the system increases and decreases in a quasi-static manner.
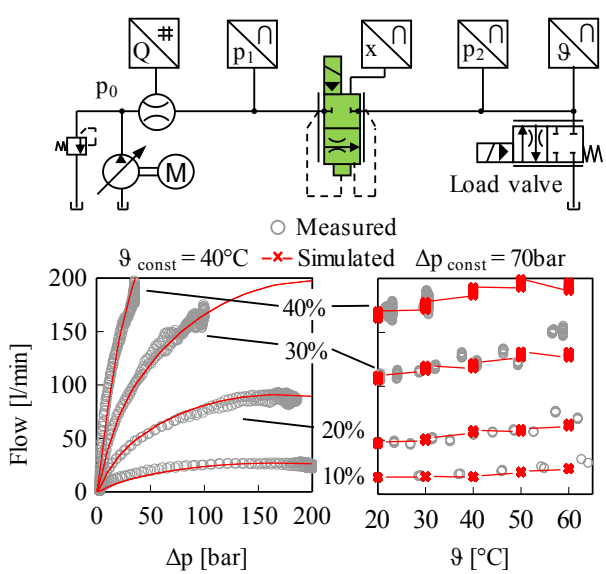

Figure 6: Experimental setup (top) / simulated and measured steady state flow behaviour for discrete input-signals $U_{\text {rel }}$ (bottom)

Figure 6 (bottom) shows a comparison of measurement data and simulation results for constant and varying temperatures. The results show a very good correlation.

\section{FLOW MAPPING OF TEST DATA}

\subsection{Training data sets}

To test the effectiveness of the approximation methods, different simulated and experimental data-sets are used, as shown in Figure 7.
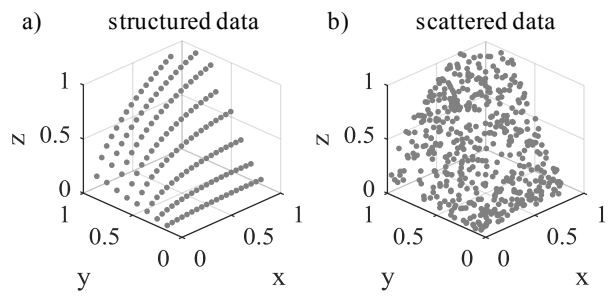

c) limited, noisy data

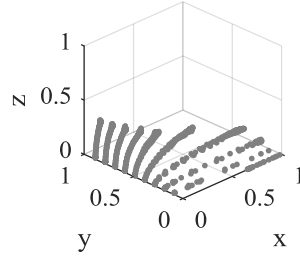

d) operating point data

Figure 7: Test source data-types a) structured data / b) scattered data / c) noisy data / d) operating point data 
Usually, structured $(\Delta p, Q)$ curves (Figure 7 a)) characterize the flow behaviour of throttle valves. These curves are determined at discrete input signals, representing the operating range. There is a high resolution along the $\mathrm{x}$-axis, whereas only a few data point exist along the y-axis. The datagap increases the requirements for the training procedures (optimization) and eventually creates great deviations between model and estimation. A comprehensive scatter data-set (Figure 7 b)) appears to be advantageous in terms of coverage. However, arbitrary data is difficult to interpret and to evaluate, which is why the use of such data is not very widespread. Limited and noisy flow curves like in Figure 7 c) are more common. The restrictions mostly result from limited capacities of the test rig or system setup. Noise is inherent to measurement data, which requires filtering of data or smoothing capabilities of the approximation procedures.

Figure 7 d) contains operating point data resulting from a typical loading cycle of a material handling excavator. The working cycle is divided in several phases, Figure 8. After picking up the bulk material (1), the operator lifts the working equipment (2) from the pile. In addition, the attachment's centre of gravity is moving closer to the rotational axis. It decreases the inertia before starting to swing of the upper carriage (3). Following the rotating phase, the working tools are positioned directly above the silo's opening (4).

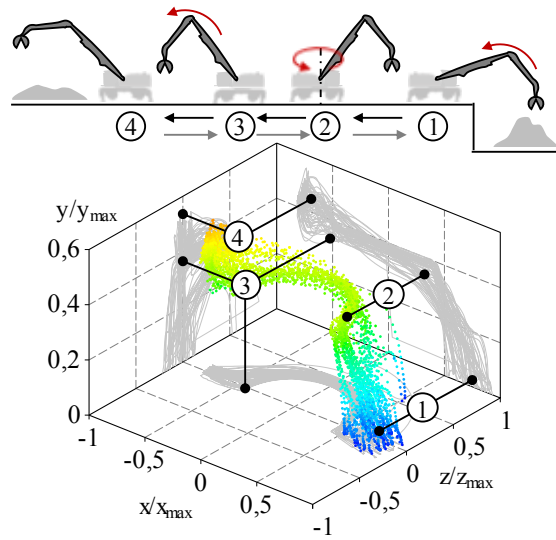

Figure 8: Loading cycle of a material handling excavator [17]

The data-point distribution represents a combination of different of upstream and downstream flow paths.

\subsection{Results and Discussion}

To investigate the quality and limitations of input-output mapping of the pilot operated seat valve, the methods proposed in ch. 3 are applied to the different training- and test-data sets (ch. 5.1) and the results are analysed. One prerequisite mentioned in the introduction was to use the flow-mapping information for control purposes. To do so, the characteristic interrelation $Q=f(\Delta p, y)$ needs to be inverted to $y=f(\Delta p, Q)$. All procedures are rechecked taking into account this premises.

In order to compare the goodness of fit for the different training-data and mapping approaches the results of the models are plotted against the data source' output values for each set of input variables. With an ideal approximation, all points would lie exactly on the $45^{\circ}$ diagonal curve. The unavoidable deviations are characterised in a box plot with regard to their dispersion. This diagram shows the median (centerline), the $25^{\text {th }}$ and $75^{\text {th }}$ percentiles (upper and lower edges of the box) as well as two extreme values, which are determined by $\mathrm{w}_{\max }=Q_{75}+1.5 \cdot I Q R$ and $w_{\min }=Q_{25}-$ $1.5 \cdot I Q R$ respectively. The range between these two extreme values covers approximately \pm 2.7 . $\sigma$ for a normally distributed random quantity and covers thus $99.3 \%$ of the realizations. All points outside these extreme values are called outliers marked separately by a "+" symbol. Additionally the relative error of the fits are plotted against the flow rate to identify critical areas. Each plot contains two different data sets. One was used to parametrize the models while the other holds previously unknown uniformly distributed data over the whole range of input values to assess the generalization capability of the different mapping approaches.

The common methods ([10x10]-gridLUT, twoLine) show a good approximation quality. However, reasonable results can only be assured for comprehensive scatter data. An extension to even more input variables requires massive additional computing effort. Furthermore, they are not designed for analytical inversion. Here recursive search algorithms of the feedforward maps have to be used.

Regarding approximation quality algebraic polynomials $\left(x^{2} y^{5}\right.$-algPoly with second order for input signal dependency of $\mathrm{x}$ and fifth order for pressure dependency of y) show similar results. They prove to be beneficial with limited and 
noisy data, though accuracy is decreasing. Using an inverse input-output-relationship for approximation also results in major deviations between model and training data. Limiting the polynomial degree to $k_{y}=2$, an analytical inversion is possible without using numerical approaches.

The extended polynomial method $([10 \times 10]-$ extPoly) is able to deal with all kinds of input data and theoretically any number of input dimensions. Regardless of the input data distribution, all results are quite close to each other, illustrating the reasonable modelling accuracy of the proposed method. Since the approach utilizes grid-point-position optimization, a weighting of different regions may be used to considerably increase local accuracy. Because the base function eq.(13) is linear, it may be rearranged in any desired way for inverse input-output-relationship, without any adverse effects.

Using artificial neural networks (MLP-ANN) the best fitting results regarding accuracy can be obtained. The fitting quality is highly dependent on the coverage of the training-data. Again, comprehensive scattered data points appear to be most beneficial. Just like all other polynomialbased approaches, ANN show very poor extrapolating behavior. To determine the inverse I/O-relationship the input and output simply have to be interchanged before training. Surprisingly, the results differ from natural $\mathrm{I} / \mathrm{O}$-assignment. Obviously, the missing distinction in the inverse pressure-flow-relationship results in major deviations between model and training data. An implementation of ANN-functions in embedded systems could be challenging, since massive floating point matrix operations are necessary.

Table 1 shows the number of parameters (coefficients) for the different methods analysed.

Table 1: Number of parameters

\begin{tabular}{ccc}
\hline \multirow{2}{*}{ Method } & \multicolumn{2}{c}{ No of parameters } \\
\cline { 2 - 3 } & $3 \mathrm{D}$ & $4 \mathrm{D}$ \\
\hline$[10 \times 10]$-gridLUT & 120 & $>1200$ \\
twoLine & 27 & - \\
$x^{2} y^{5}$-algPoly & 15 & $>35$ \\
{$[10 \times 10]$-extPoly } & 44 & 74 \\
$M L P-A N N$ & 38 & 81 \\
\hline
\end{tabular}

\section{CONCLUSION AND OUTLOOK}

Inverse, multidimensional input-output flow mapping is very important for use of valves in precision motion control applications. Due to the highly nonlinear characteristic and uncertain model structure of the cartridge valves, it is hard to formulate the modelling of their flow mappings into simple parameter estimation problems. This paper presented an analysis and comparison of different identification methods and training data sets for 3D- and 4D-flow mapping. More specifically, state of the art approaches, deep-learning methods and a newly developed approach (extPoly) had been examined. Especially ANNs and Polynomials show reasonable approximation results even for more than two inputs. However, the results are strongly dependent on the structure and distribution of the input data. Besides identification effort, the invertibility was investigated.

The next investigation steps are concerned with the further development of the proposed methods with respect to online identification. 
Figure 9: Overall result plots of proposed approximation methods for 3D- and 4D-case

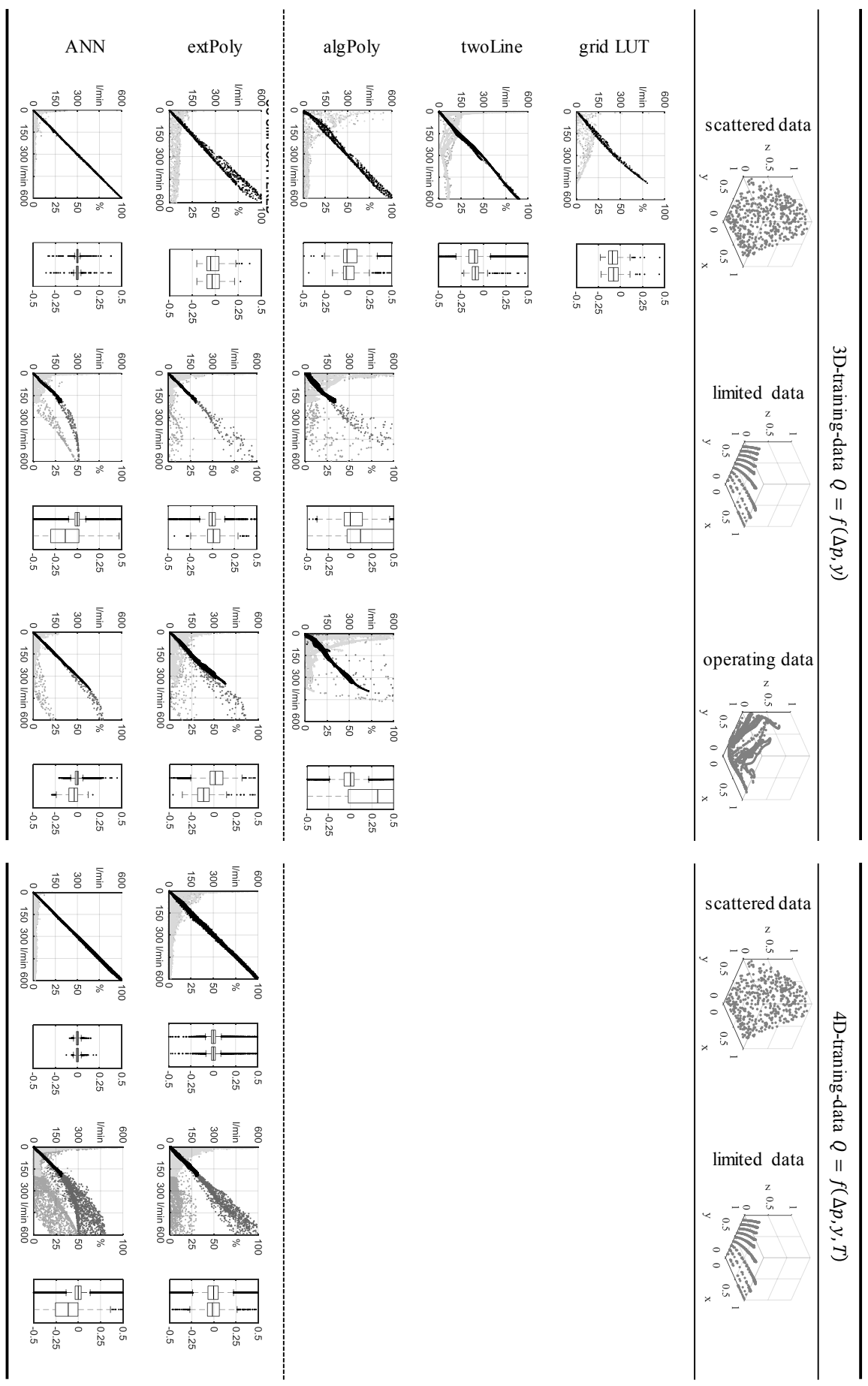




\section{NOMENCLATURE}

$\begin{array}{ll}A & \text { Area } \\ A N N & \text { Artificial Neural Network } \\ E C U & \text { Electronic Control Unit } \\ i & \text { Variable } \\ I / O & \text { Input / Output } \\ I Q R & \text { Interquartile Range } \\ M L P & \text { Multi-Layer-Perceptron } \\ M S E & \text { Mean Square Error } \\ n & \text { Number } \\ p & \text { Pressure } \\ Q & \text { Flow Rate } \\ R M S E & \text { Root Mean Square Error } \\ T & \text { Temperature } \\ V & \text { Volume } \\ x & \text { Input Variable } \\ y & \text { Input Variable } \\ z & \text { Output Variable } \\ \vartheta & \text { Temperature }\end{array}$

\section{REFERENCES}

[1] C. Bohn und H. Unbehauen, Identifikation dynamischer Systeme. Wiesbaden: Springer Fachmedien Wiesbaden, 2016.

[2] M. Vogt, N. Müller, und R. Isermann, „OnLine Adaptation of Grid-Based Look-up Tables Using a Fast Linear Regression Technique“, Journal of Dynamic Systems, Measurement, and Control, Bd. 126, Nr. 4, S. 732, 2004, doi: 10.1115/1.1849241.

[3] S. Chun, B.-R. Yoon, D.-K. Lee, und H.-M. Choi, „Correction of flow metering coefficients using two-dimensional curve fitting", in ASME-JSME-KSME 2011 Joint Fluids Engineering Conference, 2011, S. 17-25.

[4] K. Huhtala, J. K. Karen, und M. J. Vilenius, „Semi-empirical modelling of losses in components of hydrostatic transmission systems", in Design and Performance, Eigth Bath International Fluid Power Workshop, 2022.9.1995, 1996, S. 236-251.

[5] M. Gori, F. Scarselli, und Ah Chung Tsoi, „Which classes of functions can a given multilayer perceptron approximate?", in Proceedings of International Conference on Neural Networks (ICNN'96), Washington, DC, USA, 1996, Bd. 4, S. 2226-2231, doi: 10.1109/ICNN.1996.549247.

[6] C. Halfmann und H. Holzmann, Adaptive Modelle für die Kraftfahrzeugdynamik. Berlin, Heidelberg: Springer Berlin Heidelberg, 2003.
[7] P. Opdenbosch, „Auto-Calibration and Control Applied to Electro-Hydraulic Poppet Valves“, 2007.

[8] S. Liu und B. Yao, „Automated onboard modeling of cartridge valve flow mapping", IEEE/ASME Transactions on Mechatronics, Bd. 11, Nr. 4, S. 381-388, Aug. 2006, doi: 10.1109/TMECH.2006.878552.

[9] R. Isermann, Hrsg., Modellgestützte Steuerung, Regelung und Diagnose von Verbrennungsmotoren. Berlin, Heidelberg: Springer Berlin Heidelberg, 2003.

[10] O. Nelles und A. Fink, „Grid-Based LookUp Table Optimization Toolbox", IFAC Proceedings Volumes, Bd. 33, Nr. 15, S. 839844, Juni 2000, doi: 10.1016/S14746670(17)39857-9.

[11] W. S. McCulloch und W. Pitts, ,A logical calculus of the ideas immanent in nervous activity“, Bulletin of Mathematical Biophysics, Bd. 5, Nr. 4, S. 115-133, Dez. 1943, doi: 10.1007/BF02478259.

[12] R. Kruse, C. Borgelt, F. Klawonn, C. Möwes, G. Ruß, und M. Steinbrecher, Computational Intelligence: Eine methodische Einführung in Künstliche Neuronale Netze, Evolutionäre Algorithmen, Fuzzy-Systeme und Bayes-Netze. Wiesbaden: Vieweg+Teubner Verlag / Springer Fachmedien Wiesbaden $\mathrm{GmbH}$, Wiesbaden, 2012.

[13] R. Rojas, Theorie der neuronalen Netze. Berlin, Heidelberg: Springer Berlin Heidelberg, 1993.

[14] D. J. C. MacKay, „A Practical Bayesian Framework for Backpropagation Networks", Neural Computation, Bd. 4, Nr. 3, S. 448-472, Mai 1992, doi: 10.1162/neco.1992.4.3.448.

[15] B. Andersson, „On the valvistor a proprtional controlled seat valve“, Department of Mechanical Engineering, Linköping University, 1984.

[16] E. Prasetiawan, R. Zhang, und A. Alleyne, „Fundamental performance limitations for a class of electronic two-stage proportional flow valves", in American Control Conference, 2001. Proceedings of the 2001, 2001, Bd. 5, S. 3955-3960.

[17] A. Sitte, J. Weber, Uhlmann, Jonas, B. Meitinger, und Weidner, Yannick, ,MODEL BASED EFFICIENCY ANYLYSIS OF MOBILE HYDRAULIC MACHINERY (On The Example of Material Handling Machines)“, in Proceedings of the JFPS International Symposium on Fluid Power, Fukuoka, 2017. 